\title{
SUR UN THÉORÈME SPECTRAL ET SON APPLICATION AUX NOYAUX LIPCHITZIENS
}

\author{
HUBERT HENNION
}

(Communicated by George C. Papanicolaou)

\begin{abstract}
The aim of this note is to point out that the Ionescu Tulcea and Marinescu theorem can be reinforced, using a Nussbaum formula for the essential spectral radius of an operator. In this stronger version, this theorem is suitable for the spectral analysis of lipschitzian, positive, not necessarily markovian kernels, Ruelle theorem follows. As an application to Probability Theory, a large deviation theorem is proved.
\end{abstract}

Dans ce qui suit $(L,\|\cdot\|)$ est un espace de Banach complexe et $T$ un opérateur borné de $L$ de rayon spectral $\rho(T)$, la restriction de $T$ au sousespace stable $G$ est désignée par $T_{G}$.

Diverses études telles que, en Probabilités, celle des chaînes à liaisons complètes [3] et des chaînes liées à l'action de matrices aléatoires [10, 7], en Théorie Ergodique, celle de l'opérateur de Perron-Frobenius associé à une transformation dilatante $[9,5]$ et, en Mécanique Statistique, celle de l'opérateur de Ruelle [13], nécessitent une analyse spectrale d'opérateurs ayant la propriété $(D F(r))$ suivante.

Définition. On dit que $T$ a la propriété $\operatorname{DF}(r)$, s'il existe une norme $|\cdot|$ sur $L$ telle que

(i) $T$ est compact de $(L,\|\cdot\|)$ dans $(L,|\cdot|)$,

(ii) pour tout $n, n \in \mathbb{N}$, il existe des réels positifs $R_{n}, r_{n}$, tels que $\liminf _{n}\left(r_{n}\right)^{1 / n}=r<\rho(T)$ et, pour tout $f \in L,\left\|T^{n} f\right\| \leq R_{n}|f|+r_{n}\|f\|$.

La suite $\left(R_{n}\right)_{n}$ n'est soumise à aucune condition. Quand, comme il est fréquent, $T$ est un opérateur borné de $(L,|\cdot|)$ satisfaisant à (i) et vérifiant l'inégalité (ii) pour un entier $s, s \geq 1$, avec une constante $r_{s}$ telle que $r_{s}^{1 / s}<\rho(T), T$ à la propriété $\left(D F\left(r_{s}^{1 / s}\right)\right)$. En effet, par itération, on voit que l'inégalité (ii) est satisfaite pour les puissances $T^{n s}$ de $T$ avec $r_{n s}=r_{s}^{n}$.

Les propriétés spectrales envisagées dans les travaux mentionnés ci-dessus sont commodément décrites à l'aide du rayon spectral essentiel de l'opérateur $T$. Les notions de spectre et de rayon spectral essentiels d'un opérateur ont été introduites par Browder [2], de l'étude menée par cet auteur il résulte que le rayon spectral essentiel peut être défini ainsi

Received by the editors April 20, 1991 and, in revised form, October 10, 1991.

1980 Mathematics Subject Classification (1985 Revision). Primary 60J10; Secondary 28D99.

Key words and phrases. Markov chains, limit theorems, Ruelle theorem. 
Définition. Le rayon spectral essentiel de $T, \rho_{e}(T)$, est la borne inférieure de $\rho(T)$ et des réels $r^{\prime}, 0 \leq r^{\prime}$, pour lesquels il existe des sous-espaces $F$ et $H$ de $L$ tels que

$L=F \oplus H, \quad T(F) \subset F, T(H) \subset H ;$

$1 \leq \operatorname{dim} F<+\infty$ et $T_{F}$ n'a que des valeurs propres de modules $\geq r^{\prime}$;

$H$ est fermé et $\rho\left(T_{H}\right)<r^{\prime}$. Lorsque $\rho_{e}(T)<\rho(T)$, on dit que $T$ est quasi-compact.

En adjoignant à la propriété $(D F(r))$ l'hypothèse $\sup _{n}\left|T^{n}\right|<+\infty$, Ionescu Tulcea et Marinescu [8] ont prouvé que $T$ est quasi-compact. Nous voulons faire remarquer qu'un résultat plus général et plus précis peut être obtenu par application d'une formule de calcul du rayon spectral essentiel d'un opérateur due à Nussbaum [12]. Rappelons les éléments de compréhension et d'utilisation de cette formule.

Soit $A \subset L, \Gamma(A)$ est l'ensemble des réels $\delta, \delta>0$, tels qu'il existe un recouvrement fini de $A$ par des boules de $L$ de rayon $\delta$, on pose $\gamma(A)=$ $\inf \Gamma(A)$.

$\Gamma(T)$ est l'ensemble des réels $k, k \geq 0$, tels que, pour tout $A, A \subset L$, $\gamma(T(A)) \leq k \gamma(A)$, on pose $\gamma(T)=\inf \Gamma(T)$.

Si $B_{1}$ désigne la boule unité de $L, \gamma(T)=\gamma\left(T\left(B_{1}\right)\right) \leq\|T\|$, la suite $\left(\left(\gamma\left(T^{n}\right)\right)^{1 / n}\right)_{n}$ est sous-multiplicative, on a $\rho_{\gamma}(T)=\lim _{n}\left(\gamma\left(T^{n}\right)\right)^{1 / n} \leq \rho(T)$

Le résultat de Nussbaum s'énonce

Théorème 1. $\rho_{e}(T)=\rho_{\gamma}(T)$.

Corollaire 1. Si $T$ a la propriété $D F(r)$, alors $T$ est quasi-compact et $\rho_{e}(T) \leq$ $r$.

Démonstration. Notons $\tau=\|T\|$, posons, pour $f \in L, \delta>0$,

$$
B(f, \delta)=\{g: g \in L,\|g-f\|<\delta\}, \quad D(f, \delta)=\{g: g \in L,|g-f|<\delta\}
$$

et estimons $\gamma\left(T^{s+1}\right)$.

Puisque $T\left(B_{1}\right)$ est relativement compacte dans $(L,|\cdot|)$, pour tout $\varepsilon, \varepsilon>0$, il existe $\left(f_{i}\right)_{i=1}^{n}, f_{i} \in B_{1}$, telle que, si $g_{i}=T f_{i}$,

$$
T\left(B_{1}\right) \subset \bigcup_{i=1}^{n} D\left(g_{i}, \varepsilon\right) \cap B(0, \tau)
$$

Si $g \in D\left(g_{i}, \varepsilon\right) \cap B(0, \tau)$, on a

$$
\left\|T^{s} g_{i}-T^{s} g\right\| \leq R_{s}\left|g_{i}-g\right|+r_{s}\left\|g_{i}-g\right\| \leq R_{s} \varepsilon+2 \tau r_{s},
$$

de l'arbitraire de $\varepsilon$, il vient $\gamma\left(T^{s+1}\right) \leq 2 \tau r_{s}$, d'où $\rho_{\gamma}(T) \leq \liminf _{s}\left(r_{s}\right)^{1 / s}=$ $r$.

\section{LE CAS DES NOYAUX LIPCHITZIENS}

Soit $(X, d)$ un espace métrique compact. Pour une fonction complexe $f$ définie sur $X$, on pose

$|f|_{\infty}=\sup \{|f(x)|: x \in X\}, \quad m(f)=\sup \left\{\frac{|f(x)-f(y)|}{d(x, y)}: x, y \in X, x \neq y\right\}$, 


$$
\|f\|=|f|_{\infty}+m(f) .
$$

$\mathscr{C}$ est l'espace de Banach des fonctions continues sur $X$, muni de la norme de $|\cdot|\left(|\cdot|_{\infty}\right)_{\infty}$. $\mathscr{L}$ est l'espace de Banach des fonctions $f$ sur $X$ telles que $\|f\|<+\infty$ muni de la norme $\|\cdot\|$.

Soient $G$ un ensemble d'applications de $X$ dans lui-même muni d'une structure d'espace mesuré, d'une probabilité $\mu$ et $q$ une fonction mesurable de $X \times G$ dans $\mathbb{R}_{+}$telle que

$$
\sup _{g}|q(\cdot, g)|_{\infty}<+\infty, \quad \sup _{g} m(q(\cdot, g))<+\infty .
$$

Pour $f$ borélienne bornée sur $X$, on pose

$$
Q f(x)=\int q(x, g) f(g x) d \mu(g) .
$$

Par itération

$$
Q^{n} f(x)=\int q_{n}\left(x, g_{1}, \ldots, g_{n}\right) f\left(g_{n} \cdots g_{1} x\right) d \mu\left(g_{1}\right) \cdots d \mu\left(g_{n}\right),
$$

où $q_{1}=q$ et

$q_{m+n}\left(x, g_{1}, \ldots, g_{m+n}\right)=q_{m}\left(x, g_{1}, \ldots, g_{m}\right) q_{n}\left(g_{m} \cdots g_{1} x, g_{m+1}, \ldots, g_{m+n}\right)$.

Le coefficient de contraction $c\left(Q^{n}\right)$ de $Q^{n}$ est l'élément de $\mathbb{R}_{+} \cup\{+\infty\}$ défini par

$c\left(Q^{n}\right)=\sup _{\substack{x, y \in X \\ x \neq y}} \int q_{n}\left(x, g_{1}, \ldots, g_{n}\right) \frac{d\left(g_{n} \cdots g_{1} x, g_{n} \cdots g_{1} y\right)}{d(x, y)} d \mu\left(g_{1}\right) \cdots d \mu\left(g_{n}\right)$,

il est facile de vérifier que $c\left(Q^{m+n}\right) \leq c\left(Q^{m}\right) c\left(Q^{n}\right)$, on pose

$$
\bar{c}(Q)=\lim _{n} c\left(Q^{n}\right)^{1 / n} \text {. }
$$

Théorème 2. Supposons $c(Q)<+\infty$, alors $Q$ opère sur $\mathscr{C}$ et $\mathscr{L}$, son rayon spectral sur $\mathscr{C}$ est $\tilde{\rho}(Q)=\lim _{n}\left|Q^{n} 1\right|_{\infty}^{1 / n}$.

Si $\bar{c}(Q)<\tilde{\rho}(Q)$ alors $Q$ est quasi-compact sur $\mathscr{L}, \rho_{e}(Q) \leq \bar{c}(Q), \rho(Q)=$ $\tilde{\rho}(Q), \quad \rho(Q)$ est une valeur propre de $Q$ d'ordre maximal parmi les valeurs propres de module $\rho(Q)$ et il lui est associé une fonction propre positive.

Si, de plus, $Q$ est irréductible, i.e., si, pour tout $x \in X$ et $f \in \mathscr{C}$, positive, non nulle, il existe $n$ tel que $Q^{n} f(x)>0, \rho(Q)$ est la seule valeur propre de module $\rho(Q)$ et le sous-espace propre associé est engendré par une fonction strictement positive.

Démonstration. $\rho(Q), \rho_{e}(Q), \tilde{\rho}(Q)$, et $\bar{c}(Q)$ sont abrégés en $\rho, \rho_{e}, \tilde{\rho}$, et $\bar{c}$. 1 désigne la fonction constante égale à 1 sur $X$.

En posant,

$$
R_{n}=\sup \left\{m\left(q_{n}\left(., g_{1}, \ldots, g_{n}\right)\right): g_{1}, \ldots, g_{n} \in G\right\},
$$

on a

$$
\begin{aligned}
\left|Q^{n} f(x)-Q^{n} f(y)\right| \leq \int q_{n}\left(x, g_{1},\right. & \left.\ldots, g_{n}\right)\left|f\left(g_{n} \cdots g_{1} x\right)-f\left(g_{n} \cdots g_{1} y\right)\right| \\
& \times d \mu\left(g_{1}\right) \cdots d \mu\left(g_{n}\right)+R_{n} d(x, y)|f|_{\infty} .
\end{aligned}
$$


Il en résulte que $Q$ opère sur $\mathscr{C}$ et sur $\mathscr{L}$. Pour $f \in \mathscr{L}$, on a

$$
m\left(Q^{n} f\right) \leq c\left(Q^{n}\right) m(f)+R_{n}|f|_{\infty} .
$$

Il est clair que $\tilde{\rho}=\lim _{n}\left|Q^{n}\right|_{\infty}^{1 / n}=\lim _{n}\left|Q^{n} 1\right|_{\infty}^{1 / n}$, des relations $\|1\|=1$ et $|\cdot| \leq\|\cdot\|$, il résulte que $\tilde{\rho} \leq \rho$.

Dans l'action de $Q$ sur $\mathscr{L}$, on a

$$
\left\|Q^{n} f\right\| \leq\left(\left|Q^{n} 1\right|_{\infty}+R_{n}\right)|f|_{\infty}+c\left(Q^{n}\right)\|f\|,
$$

puisque la boule unité fermée de $\mathscr{L}$ est compacte pour la norme $|\cdot|_{\infty}$, les hypothèses du corollaire 1 sont satisfaites et $\rho_{e} \leq \bar{c}<\tilde{\rho} \leq \rho, Q$ est donc quasi-compact. Les valeurs spectrales de modules $\rho$ sont des valeurs propres, il en résulte que $\tilde{\rho} \geq \rho$, finalement $\tilde{\rho}=\rho$.

Il est maintenant possible de conclure en utilisant les résultats de la théorie spectrale des opérateurs positifs [15], cependant la spécificité des espaces considérés autorise une argumentation plus élémentaire.

Soit $\left(t_{n}\right)_{n}$ une suite de réels telle que $t_{n}>1$ et $\lim _{n} t_{n}=1$. On pose

$$
\phi_{n}=\left(\rho t_{n}-Q\right)^{-1} 1=\sum_{k \geq 0}\left(\rho t_{n}\right)^{-(k+1)} Q^{k} 1,
$$

$\phi_{n}$ est positive.

Considèrons la décomposition en sous-espaces fermés, $Q$-stables, $\mathscr{L}=F \oplus$ $H$, où $\operatorname{dim} F<+\infty, Q_{F}$ n'a que des valeurs propres de modules $\rho$ et $\rho\left(Q_{H}\right)<$ $\rho$.

Il existe une constante $c$ telle que, si $\lambda_{0}$ est un complexe de module $\rho$, on a

$$
\left\|\left(\lambda_{0} t_{n}-Q_{F}\right)^{-1}\right\| \leq c\left\|\phi_{n}\right\|
$$

En effet, pour tout $f \in F$,

$$
\left|\left(\lambda_{0} t_{n}-Q_{F}\right)^{-1} f\right| \leq \sum_{k \geq 0}\left(\rho t_{n}\right)^{-(k+1)} Q^{k}|f| \leq|f|_{\infty} \phi_{n},
$$

puisque $\operatorname{dim} F<+\infty$, il existe $c$ tel que, pour tout $h \in F,\|h\| \leq c|h|_{\infty}$, d'où

$$
\left\|\left(\lambda_{0} t_{n}-Q_{F}\right)^{-1} f\right\| \leq c\left|\left(\lambda_{0} t_{n}-Q_{F}\right)^{-1} f\right|_{\infty} \leq c|f|_{\infty}\left|\phi_{n}\right|_{\infty} \leq c\|f\|\left\|\phi_{n}\right\| .
$$

Soit $\lambda_{0}$ une valeur propre de module $\rho$ de $Q$, on a $\lim _{n}\left\|\left(\lambda_{0} t_{n}-Q_{F}\right)^{-1}\right\|=$ $+\infty$ donc $\lim _{n}\left\|\phi_{n}\right\|=+\infty$. Ecrivons alors $1=f+h$ où $f \in F$ et $h \in H$, on a $\phi_{n}=f_{n}+h_{n}$ avec $f_{n}=\left(\rho t_{n}-Q\right)^{-1} f$ et $h_{n}=\left(\rho t_{n}-Q\right)^{-1} h .\left(h_{n}\right)_{n}$ est bornée, en posant $c_{n}=\left\|\phi_{n}\right\|^{-1}$, on a $\lim _{n} c_{n} \phi_{n}-c_{n} f_{n}=0$, il en résulte que $\left(c_{n} f_{n}\right)_{n}$ est bornée dans l'espace de dimension finie $F$ donc relativement compacte et, par conséquent, que $\left(c_{n} \phi_{n}\right)_{n}$ est elle-même relativement compacte, en passant à la limite selon une sous-suite convenable dans l'égalité

$$
\rho t_{n}\left(c_{n} \phi_{n}\right)-Q\left(c_{n} \phi_{n}\right)=c_{n} 1,
$$

on prouve l'existence de $f,\|f\|=1, f \geq 0$ telle que $\rho f-Q f=0$.

Toutes les valeurs propres de module $\rho$ sont des pôles de même ordre de $(\lambda-Q)^{-1}$ et $\left(\lambda-Q_{F}\right)^{-1}$. Si $\lambda_{0}$ est un pôle d'ordre $k$ de $\left(\lambda-Q_{F}\right)^{-1}$, on a, pour tout $\ell \leq k-1, \lim _{n}\left(1-t_{n}\right)^{\ell}\left\|\left(\lambda_{0} t_{n}-Q_{F}\right)^{-1}\right\|=+\infty$, et, de l'inégalité précédemment établie, il vient $\lim _{n}\left(1-t_{n}\right)^{\ell}\left\|\left(\rho t_{n}-Q\right)^{-1} 1\right\|=+\infty$, de sorte que le pôle $\rho$ a une multiplicité $\geq k$. 
D'après l'hypothèse d'irréductibilité, si $Q h=\rho h$ et $h \geq 0$, on a $h>0$. L'opérateur markovien $\bar{Q}$ défini, pour $f$ borélienne bornée, par

$$
\bar{Q} f(x)=\frac{1}{\rho h(x)} Q(h f)(x),
$$

est irréductible, donc sa seule valeur propre de module 1 est 1 , elle est simple et les fonctions propres associées sont les constantes.

Corollaire 2. Soit $k$ une fonction mesurable de $X \times G$ dans $\mathbb{C}$ telle que $\sup _{g}|k(\cdot, g)|_{\infty}<+\infty, \sup _{g} m(k(\cdot, g))<+\infty$ et que $q(x, g)=|k(x, g)|$ satisfasse aux hypothèses $d u$ théorème 2 , alors l'opérateur associé $K$ opère sur $\mathscr{C}$ et sur $\mathscr{L}$ et, dans cet espace, $\rho_{e}(K) \leq \bar{c}(Q)$ et $\rho(K) \leq \rho(Q)$, où $Q$ est l'opérateur associé à $q$.

Démonstration. En reprenant les estimations précédentes, il apparait qu'il existe une constante $R_{n}^{\prime}$ telle que, pour $f \in \mathscr{L}$,

$$
\left\|K^{n} f\right\| \leq\left(\left|Q^{n} 1\right|_{\infty}+R_{n}^{\prime}\right)|f|_{\infty}+c\left(Q^{n}\right)\|f\|,
$$

d'où la première inégalité. Pour établir la seconde il suffit de considérer le cas où $\rho_{e}(K)<\rho(K)$, on a alors $\rho(K) \leq \tilde{\rho}(K)$ et l'on conclut à l'aide de $\tilde{\rho}(K) \leq \tilde{\rho}(Q)=\rho(Q)$.

Remarque. Supposons que $k(x, g)=q(x, g) \exp (i \alpha(x, g))$, où $\alpha$ est une application de $X \times G$ dans $\mathbb{R}$ satisfaisant aux conditions $\sup _{g}|\alpha(\cdot, g)|_{\infty}<$ $+\infty, \sup _{g} m(\alpha(\cdot, g))<+\infty$. Si $Q$ est irréductible, l'application à la probabilité de transition relativisée $\bar{Q}$ du lemme 3 de [5] montre que $\rho(K)=\rho(Q)$ si et seulement si il existe $u \in \mathscr{L}$ et $\theta \in \mathbb{C}$ tels que, pour tout $x \in X$, $\alpha(x, g)=u(g x)-u(x)+\theta, q(x, g) d \mu(g)$ p.p.

\section{ApPLicAtions}

Dans ce paragraphe nous illustrons l'usage qui peut être fait du théorème 2 .

Cas markovien. Si $Q$ est markovien, c'est à dire si $Q 1=1$, et si $\bar{c}(Q)<1$, l'opérateur $Q$ est quasi-compact sur $\mathscr{L}$.

La vérification de la condition $\bar{c}(Q)<1$ peut s'avérer délicate et l'on pourra tirer avantage de la démarche adoptée au paragraphe 2 de [6].

Soient $Z=\left(Z_{n}\right)_{n}$ une chaîne de Markov de probabilité de transition $Q$, $\phi$ une fonction de $\mathscr{L}$ et, pour $n \geq 1, S_{n}=\phi\left(Z_{1}\right)+\cdots+\phi\left(Z_{n}\right)$. La quasicompacité de $Q$ permet de mettre en œuvre une méthode due à Nagaev [11] et développée dans [5] pour étudier le comportement asymptotique de la suite $\left(S_{n}\right)_{n}$. Schématiquement, si $Q_{\lambda}$ est l'opérateur défini sur $\mathscr{L}$ par

$$
Q_{\lambda} f(x)=\int \exp \{\lambda \phi(g x)\} f(g x) q(x, g) d \mu(g),
$$

on a

$$
Q_{\lambda}^{n} 1(x)=E_{x}\left[\exp \left\{\lambda S_{n}\right\}\right],
$$

où $E_{x}$ est l'opérateur d'espérance pour la chaîne issue de $x$. La théorie des perturbations d'un opérateur [4] montre que, pour $|\lambda|$ suffisamment petit, $Q_{\lambda}$ est, comme $Q$, quasi-compact de sorte que le comportement asymptotique de $Q_{\lambda}^{n} 1$ est régi par la restriction de $Q_{\lambda}$ à un sous-espace de dimension finie. 
Considérons, par exemple, le cas étudié dans [10] où $G$ est le groupe des matrices $d \times d$ inversibles à coefficients réels agissant sur l'espace projectif de dimension $d-1, X$, muni de sa distance usuelle. Si la probabilité $\mu$ est fortement irréductible et contractante, on montre qu'il existe un réel $\alpha$, $0<\alpha<1$, tel que

$$
\bar{c}=\lim _{n}\left\{\sup _{\substack{x, y \in X \\ x \neq y}} \int\left(\frac{d\left(g_{n} \cdots g_{1} x, g_{n} \cdots g_{1} y\right)}{d(x, y)}\right)^{\alpha} d \mu\left(g_{1}\right) \cdots d \mu\left(g_{n}\right)\right\}^{1 / n}<1,
$$

substituant à $d$ la distance $d^{\alpha}$, on voit que l'opérateur

$$
Q f(x)=\int f(g x) d \mu(x)
$$

est quasi-compact sur $\mathscr{L}$ et que $\rho_{e}(Q) \leq \bar{c}$. L'étude des opérateurs $Q_{\lambda}$ permet de prouver un théorème de la limite centrale et un théorème des grands écarts pour la suite de variables aléatoires $\left(\log \left\|Y_{n} \cdots Y_{1} x\right\|\right)_{n}$ où $\left(Y_{n}\right)_{n}$ est une suite de matrices aléatoires indépendantes de même loi $\mu$ et $x \in \mathbb{R}^{d}$.

Cas non markovien. Certains noyaux lipchitziens apparaissant en Mécanique Statistique [13] et dans l'étude des transformations dilatantes [9] ne sont pas markoviens mais concernent un ensemble de transformations $G$ ayant la propriété

$$
\begin{aligned}
& \chi(r), \text { il existe un réel } r, 0 \leq r<1, \text { tel que, pour tout } x, y \in \\
& X, g \in G, d(g x, g y) \leq r d(x, y) .
\end{aligned}
$$

On a alors $c\left(Q^{n}\right) \leq r^{n}\left|Q^{n} 1\right|_{\infty}$ puis $\bar{c}(Q) \leq r \tilde{\rho}(Q)$. $Q$ est quasi-compact, $\rho_{e}(Q) \leq r \rho(Q)$ et, avec les notations du Corollaire 2, $\rho_{e}(K) \leq r \rho(Q)$. Dans [14] Ruelle établit ces inégalités par une autre méthode.

Pour illustrer ce résultat et décrire plus précisément la méthode esquissée au paragraphe précédent, revenons au cas $Q$ est markovien en conservant l'hypothèse $\chi(r)$. On a, pour tout $\lambda \in \mathbb{R}, \rho_{e}\left(Q_{\lambda}\right) \leq r \rho\left(Q_{\lambda}\right)$. Ceci permet, comme nous allons le montrer, d'établir un théorème des grands écarts dans lequel la condition de localisation du paramètre, ci-après noté $a$, est précisée, ce qui ne peut être fait lorsqu'un argument de la théorie des perturbations est utilisé. Comme la technique mise en œuvre s'applique de façon naturelle au cas, plus général, où $\phi$ est fonction de deux variables, nous allons nous placer dans ce cadre.

On suppose $Q$ irréductible.

Puisque $X$ est compact, $Q$ a une probabilité invariante, d'après le théorème 2 , une telle probabilité est uniquement déterminée sur $\mathscr{L}$, donc, il n'en existe qu'une, désignons la par $\nu$.

Soit maintenant $\phi$ lipschitzienne sur $X \times X$ telle que

(i) $\gamma=\int \phi(x, g x) q(x, g) d \mu(g) d \nu(x)=0$,

(ii) il n'existe pas de fonction $u \in \mathscr{L}$ telle que, pour tout $x \in X$,

$$
\phi(x, g x)=u(g x)-u(x), \quad q(x, g) d \mu(g) \text { p.s. }
$$

Pour $\lambda \in \mathbb{R}$ et $f \in \mathscr{L}$, on pose

$$
Q_{\lambda} f(x)=\int \exp \{\lambda \phi(x, g x)\} f(g x) q(x, g) d \mu(g) .
$$


Théorème 3. $Q_{\lambda}$ est un opérateur borné de $\mathscr{L}$ et, si $\rho(\lambda)$ désigne son rayon spectral, $\log \rho$ est une fonction analytique strictement convexe sur $\mathbb{R}$ et telle que $(\log \rho)(0)=(\log \rho)^{\prime}(0)=0$.

Soit $P_{x}$ la loi de la chaîne de Markov $\left(Z_{n}\right)_{n \geq 0}$ de probabilité de transition $Q$ issue de $x \in X$, alors, pour $0<a<a_{\infty}$,

$$
\lim _{n} \frac{1}{n} \log P_{x}\left(\left[\phi\left(Z_{0}, Z_{1}\right)+\cdots+\phi\left(Z_{n-1}, Z_{n}\right)>n a\right]\right)=-c(a),
$$

où $c(a)=\sup \{\lambda a-\log \rho(\lambda): \lambda \in \mathbb{R}\}$ et $a_{\infty}=\lim _{+\infty} \lambda^{-1} \log \rho(\lambda)$.

Démonstration. $Q_{\lambda}$ satisfait aux hypothèses du théorème 1 et est irréductible donc il a $\rho(\lambda)>0$ pour valeur propre simple associée à une fonction propre strictement positive $h_{\lambda} \in \mathscr{L}$ et il existe un projecteur $\Pi_{\lambda}$ sur le sous-espace engendré par $h_{\lambda}$ qui commute à $Q_{\lambda}$ et tel que $Q_{\lambda}\left(I-\Pi_{\lambda}\right)$ ait un rayon spectral $<\rho(\lambda)$.

La théorie des perturbations [4] montre que $\rho$ est analytique. D'après [5], les dérivées première et seconde de $\rho$ s'interprètent à l'aide de la probabilité de transition $P_{\lambda}$ définie par

$$
P_{\lambda} f(x)=\frac{1}{\rho(\lambda) h_{\lambda}(x)} \int \exp \{\lambda \phi(x, y)\} f(y) h_{\lambda}(y) Q(x, d y) .
$$

$P_{\lambda}$ a, comme $Q$, une probabilité invariante unique $\nu_{\lambda}$ et on a

$$
\rho^{-1}(\lambda) \rho^{\prime}(\lambda)=\gamma_{\lambda}, \quad \rho^{-1}(\lambda) \rho^{\prime \prime}(\lambda)=\gamma_{\lambda}^{2}+\sigma_{\lambda}^{2},
$$

le réel $\sigma_{\lambda}^{2}$ ne peut être nul que dans le cas où il existe une fonction $u \in \mathscr{L}$ telle que $\phi(x, y)=u(y)-u(x), \nu_{\lambda}(d x) P_{\lambda}(x, d y)$ p.s., puisque le support de $\nu_{\lambda}$ est $X$ et que $P_{\lambda}(x, \cdot)$ équivaut à $Q(x, \cdot), \sigma_{\lambda}^{2}=0$ serait en contradiction avec (ii); on a donc $(\log \rho)^{\prime \prime}(\lambda)=\sigma_{\lambda}^{2}>0$, tandis que, d'après $(\mathrm{i}),(\log \rho)^{\prime}(0)=\gamma=0$.

Il est facile de vérifier que, pour $f \in \mathscr{L}, \Pi_{\lambda} f=\nu_{\lambda}\left(h_{\lambda}^{-1} f\right) h_{\lambda}$, de sorte que $\Pi_{\lambda} 1$ est strictement positive. En posant $S_{n}=\phi\left(Z_{0}, Z_{1}\right)+\cdots+\phi\left(Z_{n-1}, Z_{n}\right)$, on a

$$
E_{x}\left[\exp \left(\lambda S_{n}\right)\right]=Q_{\lambda}^{n} 1(x)=(\rho(\lambda))^{n} \Pi_{\lambda} 1(x)+\left[Q_{\lambda}\left(I-\Pi_{\lambda}\right)\right]^{n} 1(x),
$$

et, par suite, $\lim _{n} \frac{1}{n} \log E_{x}\left[\exp \left(\lambda S_{n}\right)\right]=\log \rho(\lambda)$, les propriétés de $\log \rho$ permettent de conclure en appliquant un énoncé classique de la théorie des grands écarts (voir, par exemple, [1, chapitre $\mathrm{V}$, lemme 6.2]).

\section{REFERENCES}

1. P. Bougerol et J. Lacroix, Products of random matrices with applications to Schrödinger operators, Progr. Probab. Statist., Birkhäuser, Boston, 1985.

2. F. E. Browder, On the spectral theory of elliptic differential operators, Math. Ann. 142 (1961), 22-130.

3. W. Doeblin et R. Fortet, Sur les chaînes à liaisons complètes, Bull. Soc. Math. France 65 (1937), 132-148.

4. N. Dunford et J. T. Schwartz, Linear operators. Part I, Pure Appl. Math., Vol. VII, Interscience, New York, 1967.

5. Y. Guivarc' $\mathrm{h}$ et J. Hardy, Théorèmes limites pour une classe de chaînes de Markov et applications aux difféomorphismes d'Anosovi, Ann. Inst. H. Poincaré 24 (1988), 73-98.

6. Y. Guivarc' $h$ and A. Raugi, Products of random matrices: convergence theorems, Contemp. Math., vol. 50, Amer. Math. Soc., Providence, RI, 1986, pp. 31-54. 
7. H. Hennion, Dérivabilité du plus grand exposant caractéristique des produits de matrices aléatoires indépendantes à coefficients positifs, Ann. Inst. H. Poincaré 27 (1991).

8. C. T. Ionescu Tulcea et G. Marinescu, Théorie ergodique pour des classes d'opérateurs non complètement continus, Ann. of Math. (2) 52 (1950), 140-147.

9. G. Keller, Generalized bounded variation and applications to piecewise monotonic transformations, Z. Wahrsch. Verw. Gebiete 69 (1985), 461-478.

10. E. Le Page, Théorèmes limites pour les produits de matrices aléatoires, Probability Measures on Groups, Proceedings Oberwolfach, Lectures Notes Series in Math., vol. 928, 1982, pp. 258-303.

11. S. V. Nagaev, Some limit theorems for stationnary Markov chains, Theory. Probab. Appl. 2 (1957), 378-406.

12. R. D. Nussbaum, The radius of essential spectrum, Duke Math. J. 37 (1970), 473-478.

13. D. Ruelle, Thermodynamic formalism, Encyclopedia Math. Appl., vol. 5, Cambridge Univ. Press, Cambridge and New York, 1978.

14. _ An extension of the theory of Fredholm determinants, Inst. Hautes Étude Sci. Publ. Math. 72 (1990).

15. H. H. Schaefer, Topological vector spaces, Macmillan Series in Advanced Mathematics and Theoretical Physics, Macmillan, New York, 1966.

Institut de Recherche Mathématique de Rennes, Université de Rennes I, Campus de Beaulieu, 35042 Rennes Cedex, France 